\title{
Gene expression profiling of oral submucous fibrosis using oligonucleotide microarray
}

\author{
YANJIA HU ${ }^{1}$, XINCHUN JIAN ${ }^{1}$, JIEYING PENG $^{2}$, XIAOWEN JIANG ${ }^{1}$, NING $^{1}{ }^{1}$ and SHANGHUI ZHOU ${ }^{1}$ \\ ${ }^{1}$ Departments of Oral and Maxillofacial Surgery, and ${ }^{2}$ Department of Oral Medicine, \\ Xiang Ya Hospital, Central South University, 410008, Changsha P.R. China
}

Received March 26, 2008; Accepted May 8, 2008

DOI: 10.3892/or_00000005

\begin{abstract}
Although oral submucous fibrosis (OSF) is the most common precancerous lesion of the oral cavity in Southeast Asia where the habit of betel quid (BQ) chewing is popular, its molecular biological properties are largely unknown. The aim of this study was to identify the genes responsible for its pathogenesis and malignant transformation using oligonucleotide microarray. The expression profiles of 14,500 genes in human oral submucous fibrosis and normal control were analyzed using Affymetrix U133A 2.0 GeneChip arrays. The results revealed that 716 genes were upregulated and 149 genes were downregulated in OSF. Hierarchical clustering revealed that the gene expression profiles of normal and OSF were clearly distinct by these different expression genes. Gene Ontology (GO) and relevant bioinformatics tools identified a list of significant differentially expressed genes involved in immune response, inflammatory response and epithelial-mesenchymal transition (EMT) induced by TGF- $\beta$ signaling pathway. Five EMT-related genes including SFRP4, THBS1, MMP2, ZO-1, and CK18 were validated with RT-PCR. Our data suggested that gene abnormalities in immune response, inflammatory response and EMT induced by TGF- $\beta$ might play an important role in the pathogenesis and malignant transformation of OSF.
\end{abstract}

\section{Introduction}

Oral submucous fibrosis (OSF) is a chronic insidious disease affecting any part of the oral cavity and sometimes the pharynx. It has been identified as a precancerous condition and the incidence of oral squamous cell carcinoma has been estimated as $7.6 \%$ in patients followed for a period of 17 years (1). There is conclusive evidence for the role of areca nut as the major risk factor in the development of OSF, but

Correspondence to: Dr X.C. Jian, Department of Oral and Maxillofacial Surgery, Xiang Ya Hospital, Central South University, No. 88, Xiangya Road, 410008, Changsha, P.R. China

E-mail: jianxinchun@yahoo.com.cn

Key words: expression profiling, oligonucleotide microarray, oral submucous fibrosis, gene ontology, pathways the mechanisms by which this occurs are not fully understood. Recently, epithelial-mesenchymal transition (EMT) has been shown to be crucial for the pathogenesis of tissue fibrosis and cancer. Emerging evidence suggests that EMT is also a crucial event in oral squamous cell carcinoma (OSCC) $(2,3)$. The study of Yang et al demonstrated the morphological changes in both NHOK and OECM-1 cells from polygonal or squamous to more spindle-shaped cells under the treatment of ANE (4). Thus, we hypothesized that EMT is vital to the pathogenesis of oral cancer and OSF (5).

Previous molecular studies on related genes have been hampered because only a few genes can be studied at any one time. Interactions between different genes or genetic pathways can be overlooked. Gene expression profiling is a powerful tool to identify and target markers associated with disease or therapy. The simultaneous analysis of numerous messenger RNA expression patterns as well as their relationship in biologic functions enables us to understand the biological process of disease development and to identify important genes involved in this process. Such RNA-based global analyses of gene expression have led to the identification of large numbers of dysregulated genes in many different types of human diseases. However, there have been relatively few reports in $\operatorname{OSF}(6,7)$.

In this study, we studied the gene expression profiles of human OSF and normal buccal mucosa tissues by using the Affymetrix U133A 2.0 chips to identify genes associated with the pathogenesis and malignant transformation of OSF. We analyzed the genes of OSF tissues screened by microarray assays to determine the molecular pathways potentially involved in OSF pathogenesis by using several bioinformatics tools. Selected differentially expressed genes were further validated using semi-quantitative RT-PCR. The results obtained from the present study, therefore, lay the groundwork for future analysis of these potential markers/targets for clinical utility in the diagnosis, prognosis and treatment of OSF.

\section{Materials and methods}

Patients and tissue samples. Tissue samples were obtained, after informed consent, from 15 OSF patients undergoing surgical biopsy and 14 patients undergoing surgery for other disease at the Department of Oral and Maxillofacial Surgery, Xiang Ya Hospital, Central South University. The study 
Table I. Clinical characteristics of samples used in this study.

\begin{tabular}{|c|c|c|c|}
\hline Samples & Sex & Age & Histopathological diagnosis \\
\hline $1^{\mathrm{a}}$ & M & 24 & OSF (E) \\
\hline $2^{\mathrm{a}}$ & M & 36 & OSF (I) \\
\hline $3^{\mathrm{a}}$ & M & 22 & OSF (I) \\
\hline $4^{a}$ & M & 36 & OSF (A) \\
\hline 5 & M & 41 & OSF (A) \\
\hline 6 & $\mathrm{M}$ & 30 & OSF (I) \\
\hline 7 & M & 54 & OSF (E) \\
\hline 8 & $\mathrm{M}$ & 40 & OSF (I) \\
\hline 9 & M & 36 & OSF (I) \\
\hline 10 & M & 26 & OSF (I) \\
\hline 11 & M & 16 & OSF (A) \\
\hline 12 & M & 26 & OSF (E) \\
\hline 13 & M & 27 & OSF (I) \\
\hline 14 & M & 32 & OSF (I) \\
\hline 15 & M & 40 & OSF (I) \\
\hline $16^{\mathrm{a}}$ & M & 16 & Normal \\
\hline $17^{\mathrm{a}}$ & M & 20 & Normal \\
\hline $18^{\mathrm{a}}$ & M & 17 & Normal \\
\hline $19^{\mathrm{a}}$ & M & 30 & Normal \\
\hline 20 & M & 48 & Normal \\
\hline 21 & M & 22 & Normal \\
\hline 22 & M & 22 & Normal \\
\hline 23 & M & 49 & Normal \\
\hline 24 & $\mathrm{M}$ & 58 & Normal \\
\hline 25 & $\mathrm{M}$ & 47 & Normal \\
\hline 26 & M & 16 & Normal \\
\hline 27 & M & 16 & Normal \\
\hline 28 & M & 50 & Normal \\
\hline 29 & M & 15 & Normal \\
\hline
\end{tabular}

aSamples were studied using oligonucleotide microarrays. The other samples were used in the RT-PCR assay. Histopathological diagnosis of OSF in three stages: early (E), intermediate (I) and advanced (A) stages.

protocol was approved by the ethics committees of Central South University. All patients had palatability of chewing areca nuts and the cases who had previous local treatment for the oral mucosa or had systemic diseases (scleroderma, diabetes) were excluded. Patients were diagnosed histopathologically as OSF and staged according to the concept of Pindborg and Sirsat (8). The clinical and histopathologic features of the subjects are shown in Table I. To decrease common confoundings, all samples were buccal mucosa of males. Immediately after surgical removal, the samples were divided to two parts. One part which included the submucous tissue was used for evaluation of the stage of OSF, the other part, which was removed of partial submucous tissue, was used for RNA extraction. Suitable samples were snap frozen in liquid nitrogen and transferred to $-86^{\circ} \mathrm{C}$ storage until RNA extraction.
RNA extraction. Total RNA was isolated from the OSF and normal buccal mucosa tissue samples using TRIzol reagent, according to the manufacturer's protocol (Invitrogen Corp., CA, USA). RNA quantity was measured by UV spectrophotometry, and quality was assessed by the $260 / 280$ ratio and $1 \%$ agarose gel electrophoresis. The tissue sample quality was strictly controlled to verify the RNA integrity before use in microarray experiments. Only samples with good RNA yield and no RNA degradation were retained for microarray experiments.

Microarray analysis. Total RNA $(5 \mu \mathrm{g})$ was used to prepare biotinylated cRNAs for hybridization using the standard Affymetrix protocol (Affymetrix, CA, USA). Briefly, RNA was converted to first strand CDNA using a T7-linked oligodeoxythymidylic acid primer (Genset, CA, USA), followed by second strand synthesis (Invitrogen). The double-stranded DNA was used as template for the in vitro transcription using MessageAmp ${ }^{\mathrm{TM}}$ II aRNA Amplification Kits (Ambion, Austin, TX, USA) yielding biotin-labeled cRNA. Following cleanup and quantifying spectrophotometry, the purified biotinylated target cRNA was fragmented into short sequences. The hybridization cocktail consisted of $15 \mu \mathrm{g}$ fragmented biotin-labeled cRNA spiked with eukaryotic hybridization control. The hybridization cocktail was first hybridized to a control microarray (Test 3) to check the cRNA integrity and to assess the system veracity. After sample quality evaluation, the human genome U133A 2.0 GeneChips (Affymetrix) were directly loaded with hybridization cocktail solution and then placed in Genechip Hybridization Oven 640 (Affymetrix) rotating at $60 \mathrm{rpm}$ at $45^{\circ} \mathrm{C}$ for $16 \mathrm{~h}$. After hybridization, the arrays were washed on Genechip Fluidics Station 450 (Affymetrix) and scanned using Genechip Scanner 3000 (Affymetrix) according to the manufacturer's procedure.

Statistical analysis and data mining. The hybridization data were analyzed using GeneChip Operating Software (GCOS 1.4). The raw signal of individual probes for the 8 arrays were normalized against the chip with median raw signal intensity using the dchip software (dChip2006). Differentially expressed genes were identified by supervised analysis with the Significance Analysis of Microarrays (SAM) software (9). Normalized expression values from dChip analysis were used for a two class paired SAM analysis. The SAM software estimated the false discovery rate and generated a q-value for each gene. Similar to a P-value, a smaller q-value indicates a more significant differential expression.

Clustering analysis was done using Gene Cluster 3.0 software and TreeView 1.6 available at http://rana.lbl.gov/ EisenSoftware.htm (10). We used hierarchical clustering with Average Linkage method and Correlation (uncentered ) as similarity matrix for the genes significantly differentially expressed between normal and OSF tissues.

Analysis of GO annotations and pathway was done using the Onto-Express a web-based tool available at http:// vortex.cs.wayne.edu/Projects. Html $(11,12)$. We compared the differentially expressed genes which were identificated by SAM analysis. Significance was assessed by using the 
Table II. Primers used in the semi-quantitative RT-PCR and products.

Gene names

Primers

RT-PCR product (base pair)

\begin{tabular}{llc}
\hline CK18 & F: 5'-TAGAGTCAAGTATGAGACAGAGCTGG-3' & 400 \\
& R: 5'-TGTGAGCGTCGTCTCAGCA-3' & \\
MMP2 & F: 5'-AAGCTCATCGCAGATGCCT-3' & 570 \\
& R: 5'-CCACCAGTGCCCTCTTGA-3' & \\
SFRP4 & F: 5'-GCCCCAGAAGATTTCTTCCT-3' & 347 \\
& R: 5'-TACATGGCACAGAAGAAGAAGC-3' & \\
ZO-1 & F: 5'-ACTTAATCTAAATGGAACCACTCTATCA-3' & 295 \\
THBS1 & R: 5'-CTCAAATGCACCCCCATTACT-3' & \\
& F: 5'-AAGTGCAACTACCTGGGCCACTAT-3' & 472 \\
GAPDH & R: 5'-CATTGTAGACGTACTGGCAGTTGT-3' & \\
& F: 5'-ACCACAGTCCATGCCATC-3' & 452 \\
\hline
\end{tabular}

F, forward, R, reverse.

hypergeometric distribution, and P-values were corrected for multiple hypothesis, controlling FDR (false discovery rate). These probe set lists can be used with the Onto-Express tool, allowing one to browse through the GO terms organized in the tree structure.

Semi-quantitative RT-PCR. Total RNA $(1.5 \mu \mathrm{g})$ from normal or OSF samples was reverse transcribed, using the RevertAid First Strand cDNA Synthesis Kit (Fermentas, Vilnius, Lithuania) with an oligo-(dT) primer, according to the manufacturer's directions. The sequence of the primer and expected product sizes for the 6 genes are listed in Table II. PCR was performed in a total volume of $25 \mu \mathrm{l}$, containing $12.5 \mu \mathrm{l}$ PCR Master Mix (Fermentas), $2 \mu 1 \mathrm{cDNA}$ and $10 \mathrm{pmol}$ of primer. It ran for 30-35 cycles of denaturation, annealing, and extension each at $94^{\circ} \mathrm{C}$ for $30 \mathrm{sec}, 50^{\circ} \mathrm{C}$ or $54^{\circ} \mathrm{C}$ for $30 \mathrm{sec}$ and $72^{\circ} \mathrm{C}$ for $40 \mathrm{sec}$. The amounts of cDNA generated from each sample were confirmed by comparison to the expression levels of the housekeeping gene glyceraldehyde3-phosphate dehydrogenase (GAPDH), which served as an internal control. The PCR products were electrophoresed on a $1.5 \%$ agarose gel and visualized with ethidium bromide. Signals were captured with the Multi Genus Bio Imaging System and signal intensity was analyzed by the GeneTools software (Syngene, Synoptics Ltd., Cambridge, UK). The band intensities of each gene were normalized with those of corresponding GAPDH bands. Statistical analysis of RT-PCR intensity data was performed using Student's t-test. P-values $<0.05$ were regarded as statistically significant. SPSS software was used for statistical analysis.

\section{Results}

Differential expression of genes in OSF tissues. We analyzed the expression of 18,400 transcripts using Affymetrix U133A 2.0 microarrays for 4 OSF and 4 normal buccal mucosa samples. After probe sets with intensities absent in all samples were removed from further analysis, we obtained a final list of 15,339 probe sets for SAM analysis to identify differentially expressed genes in OSF. Using a q-value of $<5 \%$, a total of 865 significant probe sets were identified to have $>2$-fold change between the OSF and normal buccal mucosa tissues. There were 716 upregulated and 149 downregulated probe sets in OSF.

Cluster analysis. To confirm the SAM analysis result, the 8 samples were grouped using hierarchical clustering with the 865 probe sets that identified the differential expression genes in OSF. The resulting expression map was visualized with Treeview 1.6 using the average linkage clustering algorithms in the cluster 3.0 software package. As expected, 4 OSF samples clustered separately from the normal samples, meaning that the differential expression genes were able to distinguish OSF from normal tissues (Fig. 1A). The most striking subclusters were observed for immunity-related genes, mainly immunoglobulin transcripts (Fig. 1B).

Molecular characteristics of OSF. To translate the gene expression data into functional profiles, we used Onto-Express to analyze the GO terms of the differential expression genes. For each GO term, Onto-Express computes its significance (P-value), allowing one to distinguish prominent biological processes from non-significant events. We identified statistically significant biological processes that are associated with these deregulated genes. Using a P-value of $<1.0 \mathrm{E}-5$, total genes $>10$, Fig. $2 \mathrm{~A}$ lists the significant biological processes represented by these deregulated genes which include cell adhesion, immune response, cell-cell signaling, inflammatory response, cell motility, phosphate transport, transcription, skeletal development, chemotaxis, transmebrane receptor protein tyrosine kinase signaling pathway, cellular defense response and epidermis development. 


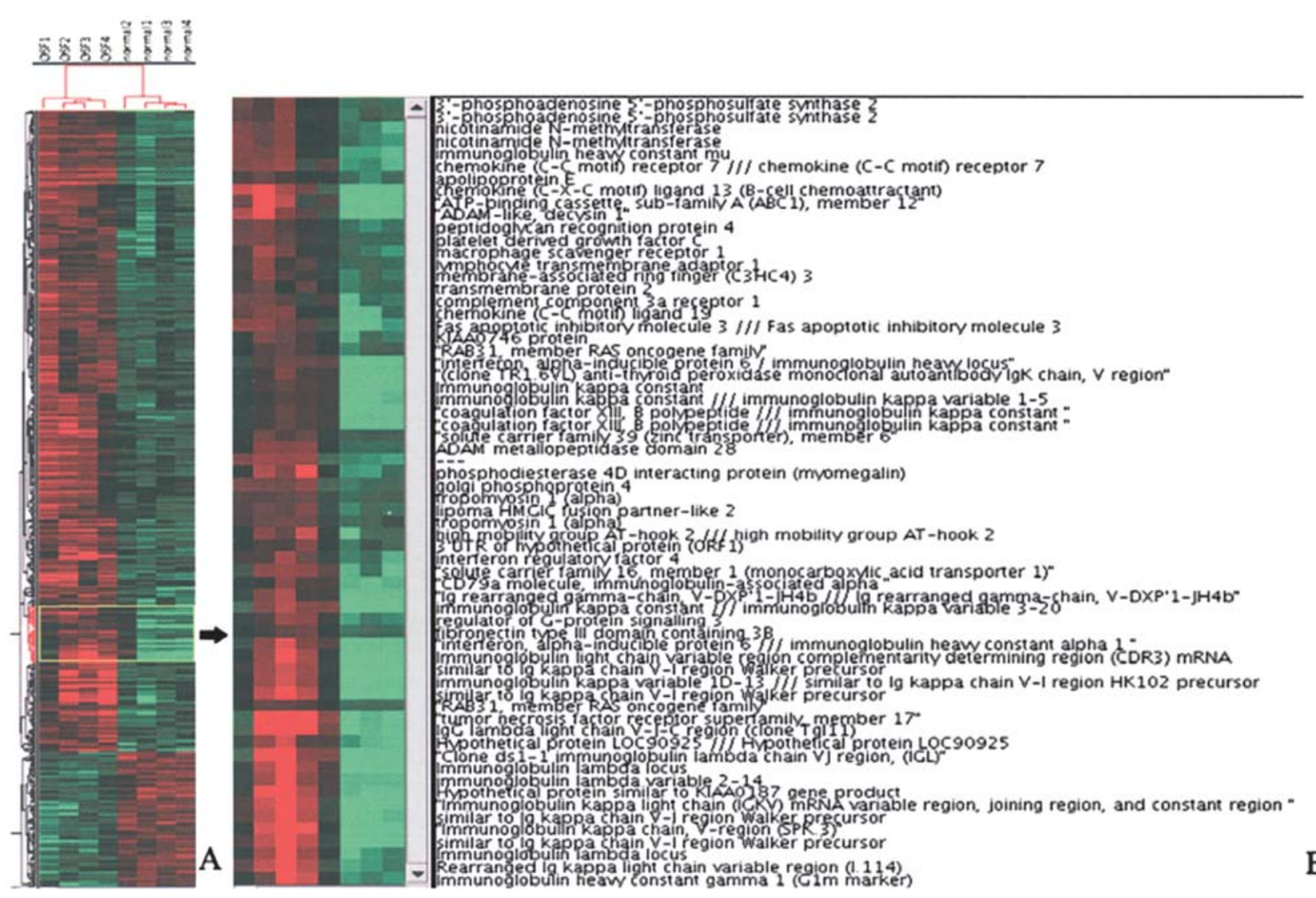

Figure 1. Cluster analysis of gene expression in OSF. (A) Hierarchical clustering of 4 OSF buccal mucosa tissues and 4 normal buccal mucosa tissues using the 865 differential gene sets. (B) The most striking subclusters were observed for immunity-related genes. Genes that are upregulated appear in red, and those that are downregulated appear in green, with the expression value reflected by the intensity of the color.

The differential expression genes were involved in various cellular functions making it difficult to determine any unifying pathways. Therefore, we applied the Onto-Pathway analysis tool to further identify signaling pathways that are associated with OSF pathogenesis. Fig. 2B shows all pathways with $\mathrm{P}$-value $<0.05$ which include antigen processing and presentation, ECM-receptor interaction, focal adhesion, cell adhesion molecules, cytokine-cytokine receptor interaction, TGF- $\beta$ signaling pathway, leukocyte transendothelial migration, phosphatidylinositol signaling system, regulation of actin cytoskeleton, complement and coagulation cascades, $\mathrm{B}$ cell receptor signaling pathway, natural killer cell mediated cytotoxity, Jak-STAT signaling pathway, type I diabetes mellitus. Indeed, GO analysis and pathway analysis described many of the major known and expected biological processes and signaling pathways that occur in the OSF. Functional analysis of selected transcripts was also done using NetAffyx (Affymetrix), and the MILANO web server for literaturebased annotation of microarray results (http://milano.md. huji.ac.il ) (13). Using the GO analysis, pathway analysis and a review of the literature, we identify and discuss the genes detected in OSF related to epithelial-mesenchymal transition (EMT) which is typically induced and maintened by TGF- $\beta$ and characterized by release of cells from epithelial polarity, remodeling of epithelial cell-cell and cell-matrix adhesion contacts and of their actin cytoskeleton (14). Genes related to focal adhesion represented the largest cluster, accounting for up to 19\% (37/194) of the modified genes. In addition, some other pathways related to EMT were located at the top in the pathway analysis according to the number of input genes (Fig. 2C), including cell adhesion molecules (22/132), regulation of actin cytoskeleton (22/206), tight junction (9/119), adhesion junction (8/77) and gap junction (6/99). The complete list of genes classified by GO and pathway is available on request. These data also showed that some genes in smooth muscle constraction and striated muscle constraction were significantly upregulated (data not shown).

Semi-quantitative RT-PCR. To validate the gene expression profiles by an independent method, semiquantitative RTPCR experiments were carried out using an independent set of $11 \mathrm{OSF}$ and 10 normal buccal mucosa tissues. Validation of our system focused on the genes related to EMT, upregulated genes associated with EMT in OSF were secreted frizzled related proteins 4 (SFRP4), matrix metalloproteinase 2 (MMP2) and thrombospondin 1 (THBS1), while downregulated genes were cytokeratin 18 (KRT18, CK18) and zona occludens 1 (ZO-1,TJP1). GAPDH was used as the internal control. As evident from the results of RT-PCR analysis (Fig. 3), the overall expression profiles for these genes correlated with the microarray-based findings. As compared to normal tissues, OSF tissues showed the enhanced expression of SFRP4 ( $\mathrm{P}=0.003)$, MMP2 $(\mathrm{P}=0.041)$ and THBS1 $(\mathrm{P}=0.036)$. Similarly, the expression of genes identified by microarray hybridization to be underexpressed was lower in OSF samples than in normal tissues. For OSF tissues, 


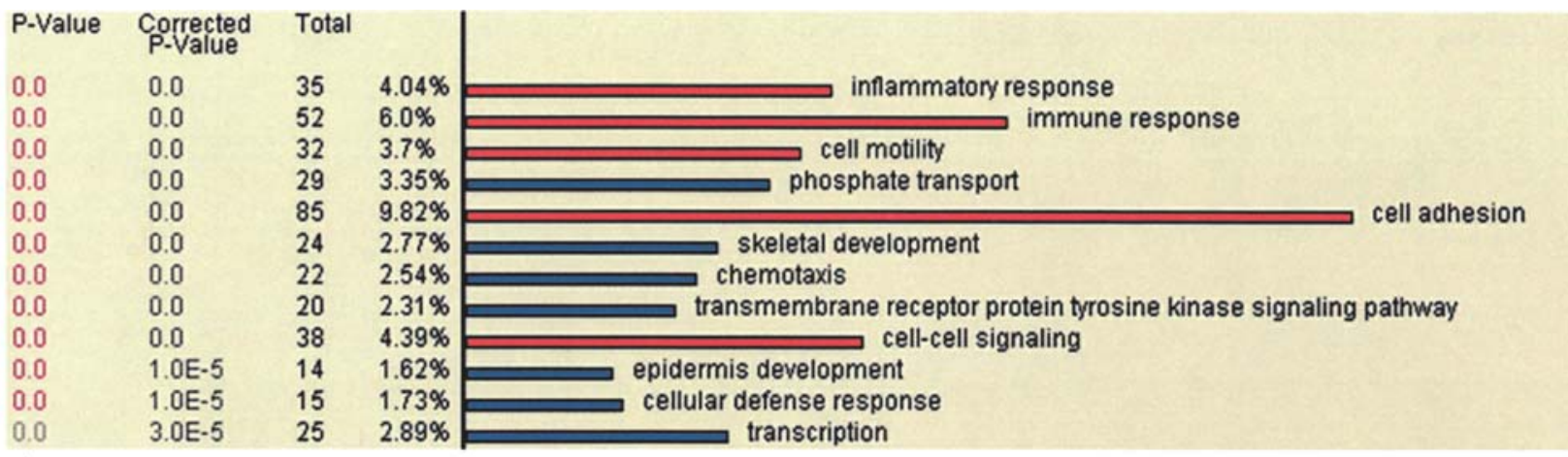

A

\begin{tabular}{|c|c|c|c|c|c|c|}
\hline \multirow{2}{*}{$\begin{array}{l}\text { Input Genes f } \\
\text { Pathway Genes }\end{array}$} & \multicolumn{2}{|c|}{ Hypergeometric } & \multicolumn{2}{|c|}{ If Analysis p-value } & \multirow[b]{2}{*}{ Impact Factor } & \\
\hline & Raw & Flag & Raw & Corrected I & & \\
\hline $10 / 86$ & $7.106 \mathrm{E}-3$ & & $0.000 E 0$ & $0.000 \mathrm{E} 0$ & 75.45 & Antigen processing and presentation - KEOQ \\
\hline $25 / 87$ & $5.576 \mathrm{E} 12$ & & 2.005E 11 & 2.005E 11 & 28.0 & ECM receptor interaction KEQG \\
\hline $37 / 194$ & $6.322 \mathrm{E}-12$ & & $2.962 \mathrm{E}-11$ & 2.962E-11 & 27.596 & Focal adhesion-KEOO \\
\hline $22 / 132$ & $1.472 \mathrm{E}-7$ & & $4.940 E-10$ & 4.940E-10 & 24.674 & Cell adhesion molecules (CAMS) - KEOO \\
\hline $35 / 256$ & 4.486E-9 & & $1.393 E-8$ & $1.393 E-8$ & 21.189 & Cytokine-cytokine receptor interaction - KEOQ \\
\hline $10 / 84$ & $6.497 \mathrm{E}-3$ & & $2.093 E-7$ & $2.093 \mathrm{E}-7$ & 18.342 & TOF-beta signaling pathway- KEOQ \\
\hline $20 / 117$ & 4.496E-7 & & 3.157E-7 & 3.157E-7 & 17.908 & Leukocyte transendothelial migration - KEOQ \\
\hline $4 / 79$ & $5.333 \mathrm{E} \cdot 1$ & & $3.282 \mathrm{E}-7$ & $3.282 \mathrm{E} \cdot 7$ & 17.867 & Phosphatidyinositol signaling system - KEOO \\
\hline $22 / 206$ & $2.298 \mathrm{E}-4$ & & $5.646 \mathrm{E}-4$ & $5.646 \mathrm{E}-4$ & 9.865 & Regulation of actin cytoskeleton - KEOQ \\
\hline $11 / 69$ & 4.995E-4 & & $1.146 \mathrm{E}-3$ & $1.146 \mathrm{E}-3$ & 9.082 & Complement and coagulation cascades - KEGQ \\
\hline $9 / 63$ & $3.372 \mathrm{E} \cdot 3$ & & $9.908 E-3$ & $9.908 \mathrm{E}-3$ & 6.649 & B cell receptor signaling pathway-KEOO \\
\hline $12 / 128$ & $1.521 \mathrm{E}-2$ & & $2.463 E-2$ & $2.463 \mathrm{E}-2$ & 5.589 & Natural killer cell mediated cytotoxicity - KEOQ \\
\hline 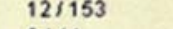 & 4.559E-2 & & $4.097 \mathrm{E}-2$ & $4.097 E-2$ & 4.984 & Jak-STAT signaling pathway - KEOG \\
\hline $6 / 44$ & $1.567 E-2$ & & $4.310 E-2$ & $4.310 E-2$ & 4.923 & Pype I diabetes mellitus - KEQG \\
\hline \multicolumn{7}{|l|}{ B } \\
\hline Input Genes I & \multicolumn{2}{|c|}{ Hypergeometric } & \multicolumn{2}{|c|}{ If Anatysis p-value } & \multirow[b]{2}{*}{ Impact Factor } & \\
\hline Pattwway Genes & Raw & Flag & Raw & Corrected & & \\
\hline $37 / 194$ & 6.322E-12 & & $2.962 \mathrm{E}-11$ & $2.962 \mathrm{E}-11$ & 27.596 & Focal adhesion-KEOO \\
\hline $35 / 256$ & $4.486 \mathrm{E}-9$ & & $1.393 \mathrm{E}-8$ & $1.393 \mathrm{E}-8$ & 21.189 & Cyokine-cyokine receptor interaction - KEOO \\
\hline $25 / 87$ & $5.576 \mathrm{E}-12$ & & 2. $005 E-11$ & 2.005E-11 & 28.0 & ECM-receptor interaction - KEOO \\
\hline 221132 & $1.472 E-7$ & & $4.940 E-10$ & $4.940 E-10$ & 24.674 & Cell adhesion molecules (CAMs) - KEOQ \\
\hline $22 / 206$ & $2.298 \mathrm{E}-4$ & & $5.646 \mathrm{E}-4$ & $5.646 \mathrm{E} \cdot 4$ & 9.865 & Regulation of actin cyoskeleton - KEOO \\
\hline $20 / 117$ & $4.496 \mathrm{E} \cdot 7$ & & 3.157E-7 & $3.157 \mathrm{E} \cdot 7$ & 17.908 & Leukocyte transendotheliat migration - KEOO \\
\hline $16 / 273$ & $2.463 \mathrm{E}-1$ & & 2.191E-1 & 2.191E-1 & 2.872 & MAPK signaling pattwway - KEQQ \\
\hline 121128 & $1.521 \mathrm{E} \cdot 2$ & & $2.463 E-2$ & $2.463 E \cdot 2$ & 5.589 & Natural kitler cell mediated cyotoxicity - KEOO \\
\hline 125153 & $4.559 E-2$ & & 4.097E-2 & $4.097 \mathrm{E}-2$ & 4.984 & Jak-STAT signaling pattwway-KEOO \\
\hline $11 / 69$ & $4.995 \mathrm{E}-4$ & & $1.146 \mathrm{E}-3$ & $1.146 \mathrm{E}-3$ & 9.082 & Complement and coagulation cascades - KEOO \\
\hline $10 / 86$ & $7.106 \mathrm{E}-3$ & & $0.000 E 0$ & $0.0000^{\circ}$ & 75.45 & Antigen processing and presentation - KEOO \\
\hline $10 / 84$ & $6.497 \mathrm{E} \cdot 3$ & & $2.093 \mathrm{E}-7$ & 2.093E-7 & 18.342 & TOF-beta signaling pathway - KEOO \\
\hline $10 / 130$ & $7.229 \mathrm{E}-2$ & & 7.297E-2 & $7.297 E-2$ & 4.282 & Axon guidance - KEOQ \\
\hline $9 / 63$ & $3.372 \mathrm{E}-3$ & & $9.908 \mathrm{E}-3$ & $9.908 E-3$ & 6.649 & B cell receptor signaling patrwray- KEOO \\
\hline $9 / 119$ & $9.985 E-2$ & & 5.327E-2 & 5.327E-2 & 4.667 & Dight junction - KEOO \\
\hline $8 / 93$ & $8.724 \mathrm{E}-2$ & & $6.140 E-2$ & $6.140 E-2$ & 4.494 & $T$ cell receptor signating patmway - KEOO \\
\hline 8177 & $3.845 E-2$ & & $6.211 \mathrm{E}-2$ & $6.211 E-2$ & 4.48 & Adherens junction-KEOQ \\
\hline $8 / 176$ & $5.993 E-1$ & & $2.516 \mathrm{E}-1$ & $2.516 \mathrm{E}-1$ & 2.684 & Calcium signating pattrway - KEOO \\
\hline 71147 & $5.070 E-1$ & & $3.853 E-1$ & $3.853 E-1$ & 2.078 & Wnt signaling pathway - KEOO \\
\hline $6 / 44$ & $1.567 \mathrm{E}-2$ & & $4.310 E-2$ & 4.310E-2 & 4.923 & Type I diabetes mellitus - KEOO \\
\hline $6 / 99$ & $3.642 \mathrm{E}-1$ & & $1.501 \mathrm{E}-1$ & 1.501E-1 & 3.372 & Gap junction - KEOO \\
\hline $6 / 72$ & $1.366 \mathrm{E}-1$ & (n) & $1.799 \mathrm{E}-1$ & $1.799 E-1$ & 3.135 & DEOF signating pathway - KEOO \\
\hline $6 / 112$ & 4.386E-1 & & 5.505E-1 & $5.505 E-1$ & 1.522 & Cell cycle - KEOO \\
\hline $6 / 302$ & $9.949 \mathrm{E}-1$ & & $6.641 \mathrm{E}-1$ & $6.641 \mathrm{E}-1$ & 1.196 & Neuroactive ligand-receptor interaction-KEOO \\
\hline
\end{tabular}

Figure 2. Functional annotation of differentially expressed genes in OSF. The length of each bar represents the number of differentially expressed genes. (A) The significant biological processes represented by these deregulated genes. [P-value of $<1.0 \mathrm{E}-5$, total genes $>10$ (blue); total genes $>30$ (red)]. (B and C) The signaling pathways that are involved in OSF pathogenesis. (B) Pathways with a P-value $<0.05$ were listed according to the P-values. (C) Pathways were listed according to the number of input genes. Those with input genes $<6$ were not listed consecutively to save space.

reduction in the expression levels was observed for CK18 $(\mathrm{P}=0.001)$ and $\mathrm{ZO}-1(\mathrm{P}=0.000)$.

\section{Discussion}

Detailed studies on differential gene expression are required to further understand the molecular pathogenesis of OSF. The ability to study the expression profile of 14,500 genes simultaneously is a powerful analytical tool that should in principle help unravel the complex molecular changes presented in the fibrosis of oral mucosa caused by chewing areca nuts. However, literature on the genomewide analysis of OSF has been extremely limited. In this study, we had to compare OSF tissue against normal buccal mucosa tissues from other individuals rather than from 'normal' mucosa of patients a distance away from the lesion site because the whole oral mucosa of patients suffered OSF. That may increase biological error due to the different social habits (drinking or smoking) between patients and controls. However, to decrease common confoundings, we did our best to make the samples used in microarrya analysis consistent with each other in age, gender, sample position, thickness and the method of sample handling. In addition, we sectioned partial submucous tissues of samples in order to make the sample consistant in thickness and to decrease the influence of submucous tissue on results. 


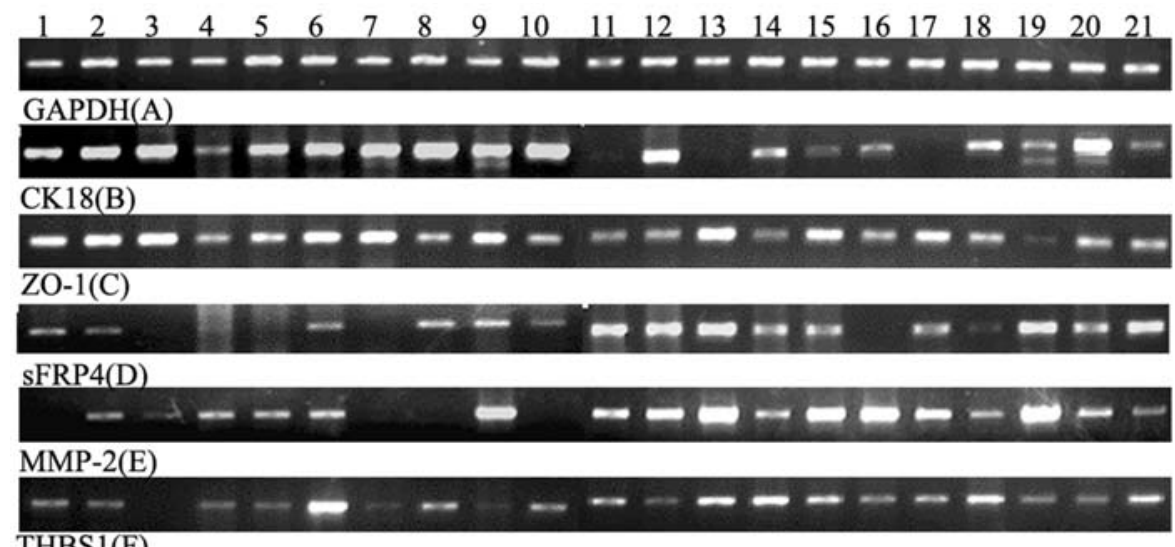

THBS1(F)

\section{$\square$ normal}

- OSF

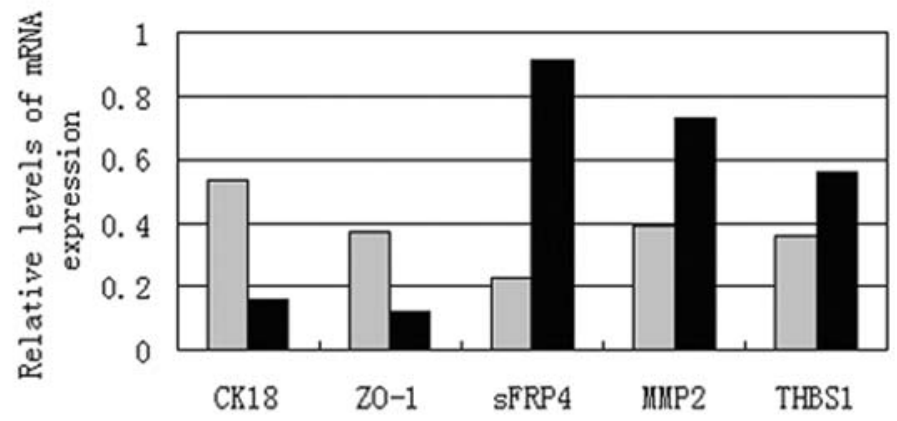

G

Figure 3. GAPDH (A), CK18 (B), ZO-1 (C), sFRP4 (D), MMP-2 (E) and THBS1 (F) reverse transcriptase-polymerase chain reaction products separated on an ethidium-staining 1.5\% agarose gel. Lanes 1-10, normal buccal mucosa; lanes 11-21, OSF buccal mucosa. (G) The level of mRNA expression of the EMTrelated genes obtained from RT-PCR. The gene expression level was normalized by using GAPDH as internal control.

In the 845 differential expression genes in OSF, some genes were previously reported by other groups using immunohistochemical assay (15-19). Some genes were not yet observed in OSF but were already identified within oral squamous cell carcinoma (OSCC) $(20,21)$. However, a number of genes had not been described in any reports on either OSF or OSCC. In the study of Kao et al (22), 18 upregulated genes and 10 downregulated genes were revealed in 1316 genes. They validated the COL I and COL III gene expression of OSF as more than the normal control, while the P450 gene expression of OSF as less than the normal controls, which was also identified in our study. However, the study of Kao et al missed many significant genes because of the limited detection capacity of the microarray used. Comparing our results with the study of Tsai et al (6), we found that a few genes represented the same trend, such as PAI-1, MMP3, STAT-1, COL4A1, TNFAIP6, CDC25B, CYP3A5, Pleiotrophin and some genes presented completely contrary trend including TIMP3, keratin 1, CDC2. Interestingly, almost all the up-regulated genes except CDC25B in growth category of OSCC shown in the study of Tsai et al had the trend to be down-regulated in OSF in our study. These genes hint at the character of the cancerization of OSF and may play an important role in the carcinogenesis of OSF.

Little is known about the molecular pathways dysregulated in OSF and knowledge of these pathways may suggest new therapeutic strategies. Therefore, we used
Onto-Express to classify genes according to functional gene ontology categories. The results of this analysis are interesting in that a number of OSF deregulated genes associated with gene ontology were defined in immune response and inflammatory response processes including antigen processing and presentation, cytokine-cytokine receptor interaction, B cell receptor signaling pathway, natural killer cell mediated cytotoxity and $\mathrm{T}$ cell receptor signaling pathway. In addition, cluster analysis identified that some immunity-related genes, mainly immuno-globulin transcripts, were significantly upregulated. The deregulation of these genes may come from infiltrated inflammatory cells. As reviewed by Chang et al (23), BQ components exhibit genotoxicity and may alter the structure of DNA, proteins and lipids, resulting in production of antigenicity. These events may provoke tissue inflammation, early cell-mediated immunity (CMI), and immune surveillance in BQ chewers. This study reconfirmed that immune response and inflammatory response are vital to the pathogenesis of OSF.

Except for immune response and inflammatory response, we observed some genes involved in smooth muscle constraction, striated muscle constraction were significantly upregulated. We supposed that EMT, a process by which epithelial cells lose cell-cell attachment, polarity and epithelial specific markers, undergo cytoskeletal remodeling and gain a mesenchymal phenotype (24), may contribute to explain this phenomenon. Although some mesenchymal markers such as vimentin (1.72), $\alpha$-smooth muscle actin (1.80) only revealed 
slight increase, some epithelial markers including CK18 (0.39), CK19 (0.06), ZO-1 (0.39) showed significant decrease. Since the role of EMT in fibrosis is evidenced in many organs $(25,26)$, numerous interesting signaling pathways from bioinformatics analysis should be further investigated to gain additional insights into the biology of OSF with regard to EMT including TGF-ß signaling pathway, Jak-STAT signaling pathway, MAPK signaling pathway, WNT signaling pathway among which crosstalk and cooperation may exist during the EMT (14). TGF- $\beta$ contributes to increased collagen level in OSF by increasing the collagen production and decreasing the collagen degradation. A recent review by Rajalalitha et al has suggested TGF- $\beta$ signaling pathway might be critical for pathogenesis of OSF (27). Many molecular events occurring in the disease via the TGF- $\beta$ pathway described in this study were identified in our study, such as COL1A2, COL3A1, COL6A1, COL6A3, COL7A1, LOX, PAT-1, TIMP1, TIMP3. However, no mention was made of EMT typically induced by TGF- $\beta$. In this study, we focus on EMT because it is a newly proposed mechanism but not well described for OSF. This study identified five genes associated with EMT. CK18 and ZO-1 as epithelial marker that are downregulated in EMT (26) were confirmed by RT-PCR $(\mathrm{P} \leq 0.001)$. CK18 that was used to evaluate the level of cell proliferation $(28,29)$ may be related with the epithelial atrophy of OSF. While protein $\mathrm{ZO}-1$ is the scaffold of tight junctions that have two functions, the barrier function and the fence function (30). We supposed that areca nut might alter the function of tight junctions by degrading the tight junctional proteins such as ZO-1. MMP-2 is critically involved in the transformation of epithelia to mesenchyme because of the ability of dissolution of the basement membrane, and MMP-2 inhibitors may be helpful in the restoration or maintenance of an epithelial phenotype $(3,31)$. The increased sFRP4 gene expression and the decreased total sFRP4 protein levels were observed in renal fibrosis induced by unilateral ureteral obstruction (UUO). Recombinant sFRP4 that can reduce Wnt signaling after renal epithelial injury may potentially suppress the expression of some EMT transcription factors to prevent epithelial de-differentiation and transition into mesenchyme (32). The role of sFRP4 in OSF is not clear, but it may possibly be further investigated as therapeutic target. TSP-1 as a protein that inhibits angiogenesis interacts with the extracellular matrix and is highly expressed in response to injuries and inflammatory reactions. The upregulation of TSP-1 may contribute to the reduction of angiogenesis in OSF. Increasing evidence shows that thrombospondin-1 is a major activator of TGF- $\beta$ in injuries or inflammatory disease (33-35). Further studies should assess whether TSP1mediated TGF- $\beta$ activation is a general spread mechanism involved in OSF.

Any attempt to understand OSF pathogenesis at the molecular level needs to take into consideration large sets of genes acting in parallel. In this study, by using a combined approach of Microarray-Bioinformatic technologies we have demonstrated some potential mechanisms contributing to OSF. The data indicate the groups of genes involved in immune response, inflammatory response and reveal EMT induced by TGF- $\beta$ singaling pathway as important components of the OSF pathogenesis. Several genes previously identified as deregulated in OSF were confirmed by our study in addition to identifying several novel genes with potential roles in EMT. Further studies will focus on confirming these findings in other aspects and exploring the contribution of individual genes in the context of the complexity revealed by this study.

\section{Acknowledgements}

We are grateful to the Cancer Research Institute and the Research Center of Molecular Biology of Central South University for their expert technical assistance. This study was supported by the National Natural Sciences Foundation of China (Grant No. 30572044).

\section{References}

1. Murti PR, Bhonsle RB, Pindborg JJ, Daftary DK, Gupta PC and Mehta FS: Malignant transformation rate in oral submucous fibrosis over a 17-year period. Community Dent Oral Epidemiol 13: 340-341, 1985.

2. Chung CH, Parker JS, Ely K, Carter J, Yi Y, Murphy BA, Ang KK, El-Naggar AK, Zanation AM, Cmelak AJ, Levy S, Slebos RJ and Yarbrough WG: Gene expression profiles identify epithelial-to-mesenchymal transition and activation of nuclear factor-\{kappa\}B signaling as characteristics of a high-risk head and neck squamous cell carcinoma. Cancer Res 66: 8210-8218, 2006.

3. Taki M, Verschueren K, Yokoyama K, Nagayama M and Kamata N: Involvement of Ets-1 transcription factor in inducing matrix metalloproteinase-2 expression by epithelial-mesenchymal transition in human squamous carcinoma cells. Int J Oncol 28: 487-496, 2006.

4. Yang SC, Lin SC, Chiang WF, Yen CY, Lin CH and Liu SY: Areca nut extract treatment elicits the fibroblastoid morphological changes, actin re-organization and signaling activation in oral keratinocytes. J Oral Pathol Med 32: 600-605, 2003.

5. Hu YJ and Jian XC: The role of epithelial-mesenchymal transition in oral squamous cell carcinoma and oral submucous fibrosis. Clin Chim Acta 383: 51-56, 2007.

6. Tsai WC, Tsai ST, Ko JY, Jin YT, Li C, Huang W, Young KC, Lai MD, Liu HS and Wu LW: The mRNA profile of genes in betel quid chewing oral cancer patients. Oral Oncol 40: 418-426, 2004.

7. Ko SY, Lin SC, Chang KW, Liu CJ, Chang SS, Lu SY and Liu TY: Modulation of KGF-1 gene expression in oral fibroblasts by ripe areca nut extract. J Oral Pathol Med 32: 399-407, 2003.

8. Pindborg JJ and Sirsat SM: Oral submucous fibrosis. Oral Surg Oral Med Oral Pathol 22: 764-779, 1966.

9. Tusher VG, Tibshirani R and Chu G: Significance analysis of microarrays applied to the ionizing radiation response. Proc Natl Acad Sci USA 98: 5116-5121, 2001.

10. Eisen MB, Spellman PT, Brown PO and Botstein D: Cluster analysis and display of genome-wide expression patterns. Proc Natl Acad Sci USA 95: 14863-14868, 1998.

11. Khatri P, Draghici S, Ostermeier GC and Krawetz SA: Profiling gene expression using onto-express. Genomics 79: 266-270, 2002.

12. Khatri P, Bhavsar P, Bawa G and Draghici S: Onto-Tools: an ensemble of web-accessible, ontology-based tools for the functional design and interpretation of high-throughput gene expression experiments. Nucleic Acids Res 32: 449-456, 2004.

13. Rubinstein R and Simon I: MILANO-custom annotation of microarray results using automatic literature searches. BMC Bioinformatics 6: 12, 2005.

14. Zavadil J and Bottinger EP: TGF-beta and epithelial-to-mesenchymal transitions. Oncogene 24: 5764-5774, 2005.

15. Tsai CH, Yang SF, Chen YJ, Chou MY and Chang YC: The upregulation of insulin-like growth factor-1 in oral submucous fibrosis. Oral Oncol 41: 940-946, 2005.

16. Yang SF, Hsieh YS, Tsai CH, Chou MY and Chang YC: The upregulation of type I plasminogen activator inhibitor in oral submucous fibrosis. Oral Oncol 39: 367-372, 2003. 
17. Utsunomiya H, Tilakaratne WM, Oshiro K, Maruyama S, Suzuki M, Ida-Yonemochi H, Cheng J and Saku T: Extracellular matrix remodeling in oral submucous fibrosis: its stagespecific modes revealed by immunohistochemistry and in situ hybridization. J Oral Pathol Med 34: 498-507, 2005.

18. Chung-Hung T, Shun-Fa Y and Yu-Chao C: The upregulation of cystatin C in oral submucous fibrosis. Oral Oncol 43: 680-685, 2007.

19. Trivedy C, Warnakulasuriya KA, Hazarey VK, Tavassoli M, Sommer P and Johnson NW: The upregulation of lysyl oxidase in oral submucous fibrosis and squamous cell carcinoma. J Oral Pathol Med 28: 246-251, 1999.

20. Liu SY, Lin MH, Yang SC, Huang GC, Chang L, Chang S, Yen CY, Chiang WF, Lee CH, Kuo YY and Liu YC: Areca quid chewing enhances the expression of salivary matrix metalloproteinase-9. J Formos Med Assoc 104: 113-119, 2005.

21. Liu CJ, Lui MT, Chen HL, Lin SC and Chang KW: MICA and MICB overexpression in oral squamous cell carcinoma. J Oral Pathol Med 36: 43-47, 2007.

22. Kao WB, Shieh YD, Hsia YJ and Shieh TY: The micro-array analysis of genetic change and proteins identification of oral submucous fibrosis. Int J Oral Max Surg 34: 139-139, 2005.

23. Chang MC, Chiang CP, Lin CL, Lee JJ, Hahn LJ and Jeng JH: Cell-mediated immunity and head and neck cancer: with special emphasis on betel quid chewing habit. Oral Oncol 41: 757-775, 2005.

24. Shook D and Keller R: Mechanisms, mechanics and function of epithelial-mesenchymal transitions in early development. Mech Dev 120: 1351-1383, 2003.

25. Kalluri R and Neilson EG: Epithelial-mesenchymal transition and its implications for fibrosis. J Clin Invest 112: 1776-1784, 2003.

26. Thiery JP and Sleeman JP: Complex networks orchestrate epithelial-mesenchymal transitions. Nat Rev Mol Cell Biol 7: 131-142, 2006.
27. Rajalalitha P and Vali S: Molecular pathogenesis of oral submucous fibrosis - a collagen metabolic disorder. J Oral Pathol Med 34: 321-328, 2005.

28. Baier PK, Baumgartner U, Wolff-Vorbeck G, Hempel S, and Hopt UT: Hepatocyte proliferation and apoptosis in rat liver after liver injury. Hepatogastroenterology 53: 747-752, 2006.

29. Kawase T, Okuda K, Yoshie H and Burns DM: Cytostatic action of enamel matrix derivative (EMDOGAIN) on human oral squamous cell carcinoma-derived SCC25 epithelial cells. J Periodontal Res 35: 291-300, 2000.

30. Sawada N, Murata M, Kikuchi K, Osanai M, Tobioka H, Kojima $\mathrm{T}$ and Chiba $\mathrm{H}$ : Tight junctions and human diseases. Med Electron Microsc 36: 147-156, 2003.

31. Cheng S, Pollock AS, Mahimkar R, Olson JL and Lovett DH: Matrix metalloproteinase 2 and basement membrane integrity: a unifying mechanism for progressive renal injury. FASEB $\mathbf{J}$ 20: 1898-1900, 2006.

32. Surendran K, Schiavi S and Hruska KA: Wnt-dependent betacatenin signaling is activated after unilateral ureteral obstruction, and recombinant secreted frizzled-related protein 4 alters the progression of renal fibrosis. J Am Soc Nephrol 16: 2373-2384, 2005.

33. Hugo C: The thrombospondin 1-TGF-beta axis in fibrotic renal disease. Nephrol Dial Transplant 18: 1241-1245, 2003.

34. Murphy-Ullrich JE and Poczatek M: Activation of latent TGFbeta by thrombospondin-1: mechanisms and physiology. Cytokine Growth Factor Rev 11: 59-69, 2000

35. Sakai K, Sumi Y, Muramatsu H, Hata K, Muramatsu T and Ueda M: Thrombospondin-1 promotes fibroblast-mediated collagen gel contraction caused by activation of latent transforming growth factor beta-1. J Dermatol Sci 31: 99-109, 2003. 